\section{LA QUÍMICA COMPUTACIONAL EN LA NUEVA FRONTERA}

\author{
Otilia Mó y Manuel Yáñez \\ Departamento de Química, Módulo 13, Facultad de Ciencias, \\ Universidad Autónoma de Madrid. \\ Campus de Excelencia UAM-CSIC. Cantoblanco, 28049-Madrid
}
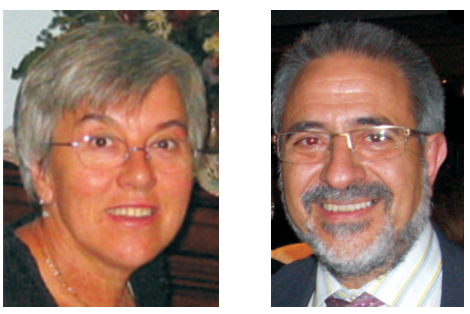

\title{
INTRODUCCIÓN
}

A lo largo de nuestra carrera profesional hemos tenido la oportunidad de asistir a más de una mesa redonda en las que expertos en el campo de la química teórica y computacional se dedicaban a hacer predicciones de cómo iba a evolucionar esta disciplina en el devenir de los años. El hecho de haber acumulado ya muchos años de actividad nos ha permitido constatar que la mayoría de las predicciones lanzadas en estos debates nunca llegaron a cumplirse, entre otras cosas porque la evolución de la metodología y sobre todo de los medios computacionales cambiaron completamente el panorama existente cuando estas profecias se hacian y aquellos expertos no fueron capaces de anticipar estos drásticos cambios. Aprendida la lección y a pesar del título de este artículo no pretendemos caer en el mismo error y hacer desde aquí profecías de cómo va a evolucionar la química teórica y computacional, pero sí queremos plantear los muchos retos a los que se enfrentará a lo largo de este siglo que está a punto de iniciar su segunda década. Sabemos que realisticamente es de todo punto imposible cubrir un objetivo tan ambicioso, queremos con ello indicar que somos conscientes que nuestro análisis se va a limitar a un número reducido de tópicos y que habrá muchos lectores a los que se les ocurrirán otros muchos, incluso más atractivos o interesantes de los que aqui pondremos sobre la palestra, pero ellos constituyen nuestra elección personal, obviamente no exenta de subjetivismo, y por tanto sujeta a cualquier crítica. Queremos también indicar que los retos que creemos se plantean en el futuro requerirán un triple esfuerzo, uno metodológico, otro en lo que los anglosajones incluirian en el mundo del software y que tiene que ver con el desarrollo de códigos más eficientes y otro tecnológico con el desarrollo de nuevas plataformas

de cálculo, que se enmarcarian en el mundo del hardware. Sería una tremenda osadía por nuestra parte hacer consideración alguna sobre estos dos últimos aspectos; pero no nos resistiremos a hacerlas respecto al primero, pero en términos que puedan ser fácilmente entendibles para los no expertos. Ojalá lo consigamos.

\section{UN POCO DE HISTORIA}

No es fácil hacer una perspectiva de futuro sin conocer un poco de la historia en este campo, ya que es difícil poder decir hacia donde vamos sin conocer bien de donde venimos. La Química Teórica y Computacional es sin duda alguna una ciencia joven, que en realidad emana de lo que inicialmente se conoció como Química Cuántica. Las denominación "teórica" es una primera generalización para englobar aquellos tratamientos no cuánticos que junto con la química cuántica permiten explorar un amplio abanico de fenómenos químicos. Un claro ejemplo de este tipo de aproximaciones (hay muchos más) es lo que se suele denominar dinámica molecular, en la que los agentes de un fenómenos químico -reactivos y productos en una reacción, por ejemplo- se describen cuánticamente, pero las trayectorias que los conectan se describen clásicamente. El calificativo "computacional" es de más reciente incorporación, y alude, como el lector ya ha adivinado al papel decisivo que los ordenadores o computadores tienen en la resolución de las complejas ecuaciones mecanocuánticas.

\section{Orígenes}

El pistoletazo de salida se dio en 1925 cuando el físico austríaco Erwin Schrödinger (Figura 1) desarrolló su fa- 


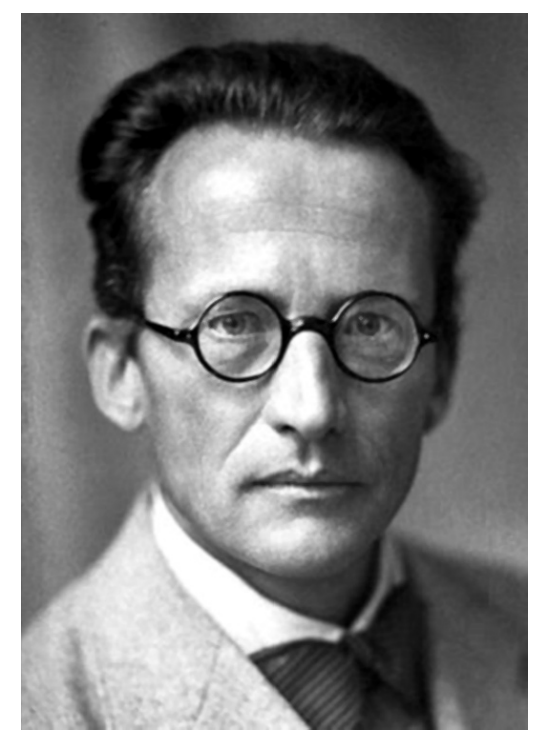

Figura 1. Erwin R. J. A. Schrödinger, con su ecuación se produjo el verdadero nacimiento de la Química Cuántica

mosa ecuación (La ec. (1) muestra su formulación más general para una dimensión, conocida como ecuación de Schrödinger dependiente del tiempo) capaz de explicar el espectro de los sistemas hidrogenoides y por tanto la cuantización de la materia sin necesidad de introducirla "a priori" como en el exitoso modelo de Bohr. Desde este momento y hasta la actualidad la evolución de la Química Cuántica no fue precisamente un camino de rosas. $\mathrm{Ni}$ siquiera sus mentes más preclaras, y estamos pensando en Paul A. M. Dirac, que fue artífice de la primera formulación relativista de las ecuaciones básicas de la Química Cuántica, eran optimistas respecto al futuro de esta rama científica. El propio Dirac afirmaría: "The underlying physical laws.... of.... the whole of chemistry are thus completely known, and the difficulty is only that the exact application of these laws leads to equations much too complicated to be soluble". En honor a la verdad, sin embargo es necesario también indicar aquí, que otra gran figura, crucial para el desarrollo de la Química Cuántica, Douglas Hartree afirmaria en 1946: It may well be that the high-speed digital computer will have as great an influence on civilization as the advent of nuclear power.

$$
\mathrm{i} \hbar \frac{\partial \Psi}{\partial \mathrm{t}}=\frac{\hbar^{2}}{2 \mathrm{~m}} \frac{\partial^{2} \Psi}{\partial \mathrm{x}^{2}}+\mathrm{V}(\mathrm{x}) \Psi(\mathrm{x}, \mathrm{t}) \equiv \hat{\mathrm{H}} \Psi(\mathrm{x}, \mathrm{t})
$$

\section{La segunda era}

El hecho de que la ecuación de Schödinger fuese sólo resoluble exactamente para sistemas monoelectrónicos parecía una barrera insalvable y condenaba a la Química Cuántica a desarrollarse en el dominio de las aproximaciones. Esta limitación de origen marcó su desarrollo y pospuso su mayoría de edad a las postrimerías del siglo XX. En efecto, no sólo era necesario desarrollar métodos matemáticos capaces de resolver aproximadamente la ecuación de Schrödinger para sistemas de varios cuerpos, era necesario calibrar la bondad y fiabilidad de los resultados por comparación con la evidencia experimental. En el primero de estos retos, las contribuciones geniales se iban sucediendo en una cascada imparable, de la mano de Hartree y de Fock (véase Figura 2) primero, y de la de Roothaan y Hall después, secundados con no menos geniales ideas de Slater, Mulliken y Pauling. Hartree, después de una visita a Cambridge de Niels Bohr en 1921, se siente compelido a usar su gran conocimiento en la solución de ecuaciones diferenciales a la solución aproximada de la ecuación de Schrödinger para varios cuerpos. En 1930 resuelve la ecuación de Schrödinger para el átomo de He, describiendo cada electrón por una función (orbital) que no depende explícitamente del movimiento instantáneo del otro electrón. Sin embargo este método no satisfacía el principio de exclusión de Pauli, para lo que es preciso expresar dicha función de onda como un determinante (genial propuesta de Slater!). Será poco después Fock quien incorpore la anti-simetrización de la función de onda al método de Hartree, dando lugar al nacimiento del método de Hartree-Fock, que proporcionaría la primera descripción cuantitativa de la estructura electrónica de átomos polielectrónicos. Lamentablemente, sin embargo, las soluciones obtenidas para la ecuación de
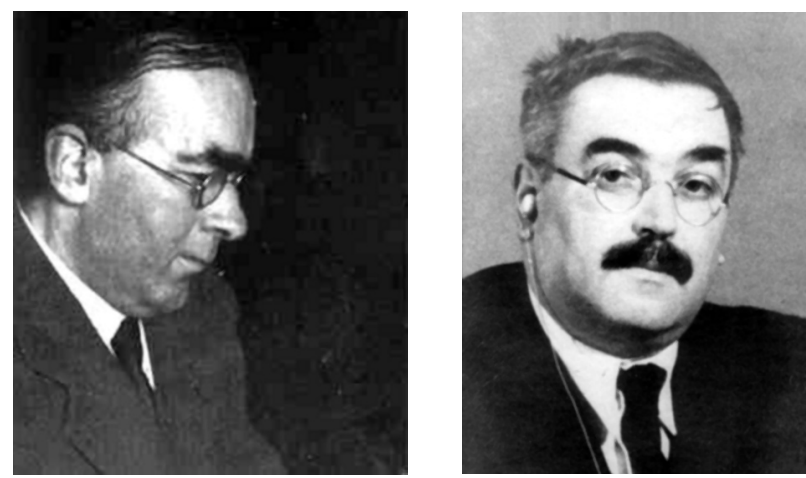

Figura 2. Douglas Hartree (izquierda) y Vladímir A. Fok. Dos figuras geniales en el desarrollo de la Química Cuántica 
Schrödinger no tienen una forma analítica cerrada, lo que limita enormemente su aplicabilidad a sistemas más y más complejos. Esta limitación sería brillantemente resuelta por Roothaan y Hall (veáse Figura 3) que proponen desarrollar los orbitales de Fock en términos de un conjunto de funciones matemáticas de forma analítica conocida (funciones de base) de manera que la búsqueda de la función de onda se reduce ahora a la determinación de los coeficientes numéricos que combinan las funciones de base para describir dicha función de onda. Nace la segunda era de la química cuántica.
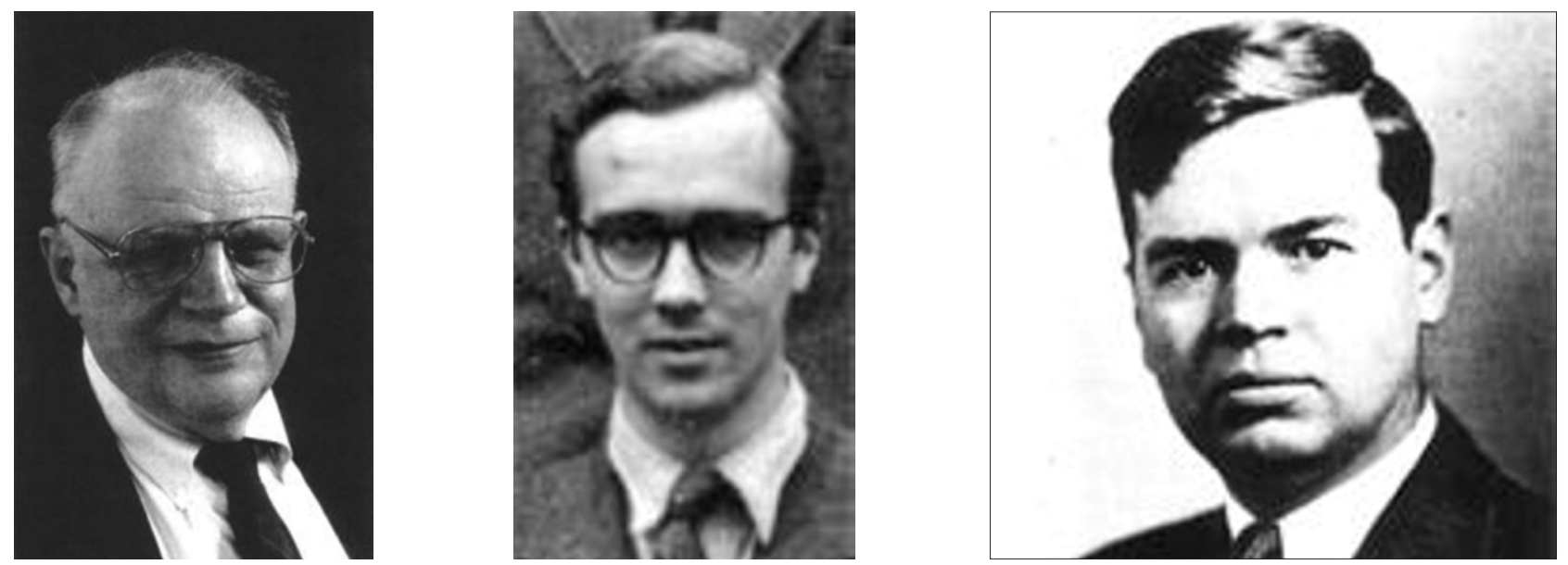

Figura 3. Clemens C. J. Roothaan (izquierda), George G. Hall (centro) y John C. Slater (derecha) desarrollaron la metodología que convertiría a la química cuántica en una herramienta capaz de tratar sistemas con muchos átomos y electrones

La posibilidad de resolver las ecuaciones de Roothaan-Hall con la precisión deseada, con tal de utilizar un conjunto de funciones de base suficientemente grande, dio origen a los Ilamados métodos ab initio, que fueron la herramienta ideal para desarrollar los primeros modelos interpretativos, que aún con conjuntos de base pequeños, eran capaces de explicar fenómenos como los efectos inductivos, hiperconjugativos o la resonancia asociada al concepto de aromaticidad, y proporcionar geometrías de sistemas para los que había una falta absoluta de información experimental. Esta nueva herramienta parecía prometedora pero estaba muy lejos de tener la precisión necesaria para convertirse en una herramienta predictiva. El método de Hartree-Fock era un modelo físico incorrecto ya que no tenía en cuenta el hecho de que el movimiento de los electrones está fuertemente correlacionado, ya que al ser partículas con carga se repelen culombianamente. Dar cuenta de la correlación electrónica no era una tarea fácil. En los cálculos ab initio HartreeFock un sistema polielectrónico, sea atómico o molecular está descrito por una única configuración electrónica y para dar cuenta de la correlación electrónica es preciso ir más allá de esta limitación incluyendo más de una configuración, en lo que se suele conocer como una interacción de configuraciones. El nuevo modelo, más riguroso en su base física proporciona resultados en mucho mejor acuerdo con la evidencia experimental, pero con la contrapartida de incrementar de un modo muy significativo el esfuerzo computacional. John A. Pople (véase Figura 4), que recibiría en 1998 el Premio Nobel de Química, por sus contribucio-

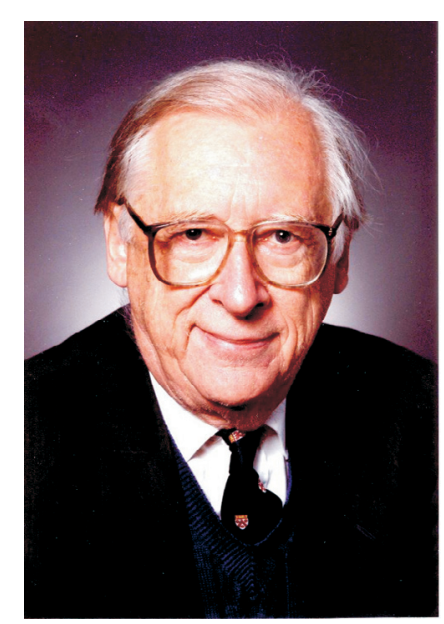

Figura 4. John A. Pople, Premio Nobel de Química en 1998 elevó a la Química Cuántica a la categoría de ciencia predictiva, y dotó a la comunidad científica en general con una potente herramienta para interpretar y predecir las propiedades de los compuestos químicos 
nes al desarrollo de la Química Cuántica, convirtiéndola en una herramienta con valor predictivo, adaptó la teoría de perturbaciones propuesta inicialmente por MØller y Plesset en los años treinta (1), para dar cuenta de la correlación electrónica. Nacía así la metodología $\operatorname{MPn}(n=2,3 \ldots 6)$ capaz de recuperar más del 90\% de la correlación electrónica a partir de funciones de onda Hartree-Fock. Se estaban poniendo los cimientos de la química cuántica moderna.

\section{La tercera era}

Todavía el único refrendo de las nuevas metodologías requería el buen acuerdo con el experimento, y por tanto los cálculos quimicocuánticos se daban siempre a posteriori de la evidencia experimental y limitando su función casi exclusivamente a la interpretación de dicha evidencia. En este hacer camino empezaron a producirse los primeros desacuerdos significativos entre la evidencia experimental y los cálculos mecanocuánticos, que la mayoría de la comunidad científica vio como una clara indicación de las muchas limitaciones que todavía afectaban a estos últimos. Pero la realidad es tozuda y pronto se pudo constatar que en más de un caso los cálculos estaban dando la respuesta correcta y era la supuesta evidencia experimental la que tenía que ser revisada. El ejemplo más paradigmático lo constituye el metileno, $\mathrm{CH}_{2}$. En 1960, Foster y Boys publican los primeros cálculos $a b$ initio sobre este sistema (2) en el que concluyen que el metileno es angular $\left(129^{\circ}\right)$ en su estado fundamental ${ }^{3} B_{1}$. Desgraciadamente, no había ninguna evidencia experimental a la sazón para refrendar estos resultados; pero un año más tarde, Herzberg concluye, tras el análisis del espectro electrónico del $\mathrm{CH}_{2}$, que el estado fundamental triplete es lineal (3). El varapalo que ello supuso para la credibilidad de los cálculos quimicocuánticos y para el prestigio personal de Foster y Boys fue enorme ${ }^{b}$. Pero afortunadamente la historia no estaba cerrada y en 1969, Harrison y Allen, mediante cálculos $a b$ initio de enlace de valencia bastante rigurosos $(4,5)$, encuentran de nuevo que el estado fundamental triplete del $\mathrm{CH}_{2}$ es angular $\left(138^{\circ}\right)$ pero no se atreven a abrir una controversia con respecto a la evidencia experimental de Herzberg. Sí lo hacen un año más tarde Bender y Schaefer que encuentran (5) en cálculos ab initio muy precisos que el $\mathrm{CH}_{2}$ tiene un ángulo de enlace de $135.1^{\circ}$ en su estado fundamental ${ }^{3} \mathrm{~B}_{1}$. Casi simultáneamente se publica un segundo estudio experimental, esta vez usando técnicas de resonancia de espín electrónico (ESR), que concluyen que el $\mathrm{CH}_{2}$ atrapado en Xenon a $4.2 \mathrm{~K}$ es angular (6). Tres meses más tarde un segundo artículo de Berheim y colaboradores ratificaba (7) que el ángulo de enlace en el $\mathrm{CH}_{2}$ era de $137.7^{\circ}$. Estas nuevas evidencias llevaron a Herzberg a re-analizar sus espectros electrónicos para concluir que estos indicaban una estructura angular para el metileno en acuerdo con los experimentos de ESR y los cálculos ab initio (8). Acabábamos de entrar en la tercera era de la Química Cuántica en la que esta disciplina pasaba de ser una herramienta cualitativa, a convertirse en una con valor cuantitativo. A esto contribuirían de un modo notable diversos factores. Uno indiscutible, la portentosa evolución de los ordenadores, cada vez más rápidos y potentes, otro, el desarrollo de metodologías ab initio de alto nivel, como el método $\operatorname{CCSD}(T)$ o las teorías $G n(n=1-4)$ de Pople y colaboradores $(9,10)$, capaces de alcanzar, lo que se vino en llamar, precisión química, ya que estos métodos son capaces de calcular magnitudes termodinámicas con una precisión de $\pm 1 \mathrm{kcal} / \mathrm{mol}$. A ello se sumó, otra herramienta poderosa, la teoría del funcional de la densidad (o DFT, density functional theory) en la que la energía se obtiene, no a través de un conocimiento de la función de onda resolviendo la ecuación de Schrödinger, sino a través de la función de densidad electrónica (11), resolviendo las ecuaciones de KohnShan (12). La gran ventaja es que mientras la función de onda es una función matemática de $3 \mathrm{~N}$ dimensiones, donde $\mathrm{N}$ es el número de electrones, la función de densidad es simplemente un función tridimensional de las tres coordenadas del espacio físico. El advenimiento de la DFT abrió la posibilidad de calcular sistemas químicos con muchos átomos y muchos electrones, inabordables, debido a su coste computacional, si se recurre a la metodología ab initio.

\section{La cuarta era}

Aunque de modo muy sucinto, en las secciones anteriores hemos pergeñado las líneas básicas sobre las que se desarroIló la química cuántica para el estudio de la estructura electrónica de átomos y moléculas (no debe olvidarse que toda la metodología hasta ahora presentada se desarrolla en el marco de la aproximación de Born-Oppenheimer de núcleos fijos en el espacio). Pero aún dentro de la aproximación de Born Oppenheimer, la energía electrónica define el potencial que gobierna el movimiento de los núcleos y por ello es posible obtener información cuantitativa sobre la rotación o la vibración de los mismos, y por ende modelizar los espectros moleculares de microondas y de infrarrojo. Típicamente, las vibraciones se obtienen en el marco de la aproximación armónica, incluyendo en el potencial sólo términos cuadráticos y suponiendo por tanto que cada oscilador cumple la ley de 
Hook. Es obvio que los osciladores reales no son armónicos y a veces son fuertemente anarmónicos, y además existe un claro acoplamiento rotación-vibración, por lo que los errores en el cálculo armónico de frecuencias suele ser de varios $\mathrm{cm}^{-1}$, lo que sitúa estas predicciones lejos de la precisión espectroscópica. Sin embargo, son muchos los científicos que consideran que hemos entrado en la cuarta era de la química cuántica, por que la extensión del potencial hasta términos cuárticos, ha permitido calcular con gran precisión los estados propios de rotación-vibración de moléculas poliatómicas y obtener frecuencias de vibración con una precisión de hasta $0.001 \mathrm{~cm}^{-1}$. Estos logros abrieron además por vez primera la posibilidad de asignar de una forma no ambigua los números cuánticos vibracionales y rotacionales de estos estados. Nos hemos dotado, pues, de una herramienta de incalculable valor para la asignación rigurosa de las bandas experimentales de absorción en infrarrojo de moléculas. Un ejemplo ilustrativo puede encontrarse en dos recientes publicaciones $(13,14)$ en las que se presenta por primera vez un análisis exhaustivo de los espectros roto-vibracionales de diferentes derivados isotópicos del agua, $\mathrm{H}_{2}{ }^{17} \mathrm{O}, \mathrm{H}_{2}{ }^{18} \mathrm{O}, \mathrm{HD}^{16} \mathrm{O}$, $\mathrm{HD}^{17} \mathrm{O}$ y $\mathrm{HD}^{18} \mathrm{O}$, que abre nuevas expectativas para un mejor conocimiento, entre otras cosas, de nuestro universo.

Este refinamiento en el cálculo de las frecuencias de vibración ha tenido también consecuencias importantísimas en el cálculo de magnitudes termodinámicas. No hay que olvidar que las energías o las entalpías experimentales incluyen la energía del punto cero, que depende directamente de las frecuencias de vibración. De poco sirve hacer un esfuerzo gigantesco tratando de incrementar la precisión de los cálculos electrónicos, si luego el error que se comete en el cálculo de la energía del punto cero es un orden de magnitud mayor que el cometido en la estimación de la energía electrónica. El notable incremento en la precisión con la que hoy en día se pueden calcular las frecuencias de vibración, ha permitido refinar de un modo significativo el cálculo de magnitudes termodinámicas y ha permitido, para poner un ejemplo relevante, anclar definitivamente la escala de afinidades protónicas absolutas combinando métodos ab initio de altísimo nivel (15), con cálculos muy precisos de las frecuencias de vibración.

\section{Algunos Retos del futuro}

El progreso científico en las últimas décadas ha sido tan espectacular y sobre todo se produce a un ritmo tal, que es de todo punto imposible hacer una recopilación ni muchos menos completa de los retos a los que la química teórica y computacional se tendrá que enfrentar en el futuro inmediato o en el futuro a medio plazo, por ello hemos pensado que lo mejor es seleccionar unos cuantos ejemplos en campos diversos para tratar de dar al lector una imagen, aunque sea difusa de la ingente tarea a la que nos enfrentamos.

Vamos a comenzar este limitado barrido por el último tema que se abordó en la sección anterior. Los retos a los que nos enfrentamos todavía en el mundo de la espectroscopía. Deciamos antes que la química cuántica ha entrado en la cuarta era en la que el tratamiento riguroso del movimiento nuclear empieza a ser posible. El desarrollo de programas como el SOFIA (Stratosferic Observatory for Infrared Astronomy) (16) (véase Figura 5) requiere el
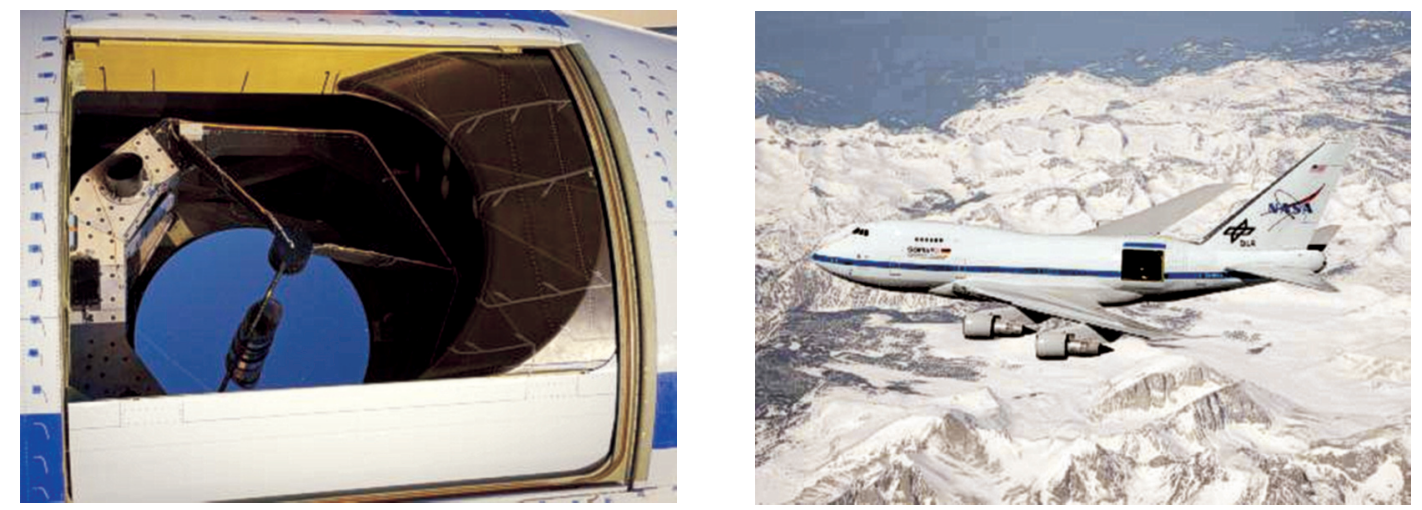

Figura 5. A la izquierda, el avión SOFIA 747SP dotado con un telescopio de infrarrojo de $2.7 \mathrm{~m}$, que se muestra en más detalle en la fotografía de la derecha 
cálculo muy preciso de espectros de infrarrojo, y lo que es más importante la asignación de los números cuánticos rotacionales y vibracionales de los estados roto-vibracionales calculados, información que resulta crucial para los espectroscopistas a la hora de intentar la asignación inambigua de las bandas de absorción experimentales. No hay que olvidar, por ejemplo que la radiación infrarroja está omnipresente en el medio interestelar y en particular en las nubes difusas. Una parte significativa de esta radiación ha sido detectada pero no asignada. Se sabe que moléculas complejas en fase gas son responsables de la emisión y que aproximadamente un 30\% de la misma es producida por hidrocarburos aromáticos policíclicos (PAH) en particular la que se detecta entre 15 y $20 \mu \mathrm{m}(17,18)$ por lo que la NASA ha puesto en marcha la NASA Ames polycyclic aromatic hydrocarbon infrared spectroscopic database, que incluye actualmente el espectro calculado para unos 600 $\mathrm{PAH}$, que pueden llegar a incluir un total de 130 átomos de carbono (19). Los resultados más precisos apuntan a que al menos parte de la radiación detectada puede provenir de especies catiónicas. Sin embargo estamos muy lejos de ser capaces de llevar a cabo una asignación completa de las emisiones observadas, y existen algunas de ellas como el pico que se detecta sistemáticamente a $6.2 \mu \mathrm{m}$ no se ha observado hasta el momento en los espectros de los $\mathrm{PAH}$ analizados.

Hay otro dominio en el que la información espectroscópica precisa es crucial y éste es el de las moléculas "evasivas", generalmente moléculas que los cálculos químico cuánticos predicen que deberían ser estables, pero que son inestables en condiciones de laboratorio, aunque sí lo son en condiciones extremas de presión y temperatura, como las que se dan típicamente en muchas zonas del espacio interestelar. Dos ejemplos típicos son los radicales NCCO $(20,21)$ y $\mathrm{HCOH}(21)$, para los que cálculos de muy alta precisión muestran la existencia de fuertes acoplamientos entre diferentes modos normales de vibración. Desgraciadamente esta situación parece ser más la norma que la excepción, lo que supone una complicación añadida a la ya complejísima tarea de la asignación de las frecuencias roto-vibracionales.

El efecto de los núcleos de un sistema molecular sobre su distribución electrónica presenta todavía otra dimensión, que se entenderá fácilmente si uno considera las sustituciones isotópicas del sistema molecular más simple, la molécula de hidrógeno. El $\mathrm{H}_{2}$ por ser homonuclear carece de momento dipolar, al igual que carece de él el $\mathrm{HD}$ en el marco de la aproximación de Born-Oppenheimer (B.0.), que ya hemos enunciado más arriba, ya que en el marco de esta formulación la energía electrónica de cualquier sistema es completamente independiente de la masa de sus núcleos; pero el HD sí tiene momento dipolar, muy pequeño ((5.85 \pm 017$\left.) 10^{-4} \mathrm{D}\right)$, pero no nulo (22). Reproducir estos sutiles efectos requiere ir un paso más allá de la aproximación de B.O., es decir requiere incluir efectos cuánticos nucleares. Ello es posible mediante una reformulación de la ecuación de Schrödinger que se ha venido en llamar aproximación de Orbitales Moleculares Nucleares (NMO) (23) y que se ha aplicado con éxito a pequeños sistemas como los complejos $\mathrm{H}_{2} \mathrm{He}^{+}$y otros (24). Es este un campo con casi infinitas posibilidades, en el que casi todo está por explorar. Los primeros resultados apuntan a que existe una relación entre la masa nuclear y la electronegatividad del átomo que sería responsable de la asimetría en la distribución electrónica del HD; pero que estamos aún muy lejos de entender. Por otro lado la aproximación NMO muestra, por ejemplo, que mientras en el complejo $\mathrm{N}_{2} \mathrm{H}_{7}{ }^{+}$el protón que mantiene unidos a las dos moléculas de amoníaco se sitúa en el punto medio entre los dos nitrógenos, la estructura del complejo $\mathrm{N}_{2} \mathrm{D}_{7}{ }^{+}$es asimétrica (vease Figura 6) (25). El origen de esta asimetría inducida por la masa del deuterio es todavía una cuestión abierta; pero son otras muchas las que están planteadas, como la quiralidad inducida por la sustitución isotópica, ya que al igual que la sustitución isotópica cambia un sistema apolar en uno polar, igualmente puede cambiar a un sistema no quiral en quiral. Es obvio que si ya de por sí la quiralidad, que surge de una violación de la paridad, conduce a enantiómeros que presentan diferencias de energía extremadamente pequeñas y por tanto muy difíciles de obtener con precisión incluso con métodos avanzados, la quiralidad por sustitución isotópica añade a esta dificultad inicial la necesidad de incluir el tratamiento cuántico de los núcleos. Todo un mundo por explorar!

Hablando de espectros moleculares conviene no olvidar que en la mayoría de los casos estos se obtienen experimentalmente en disolución, y aunque la química cuántica ha tenido avances muy significativos en el tratamiento teórico de la solvatación, los efectos del solvente en los espectros moleculares constituyen todavía un reto si se pretende tener la misma precisión que es alcanzable para 


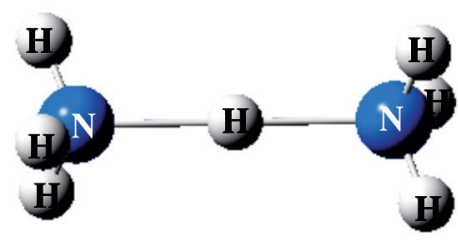

a

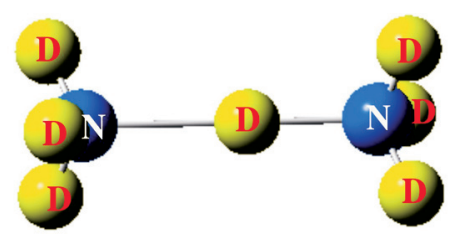

b

Figura 6. a) estructura de equilibrio del complejo $\mathrm{N}_{2} \mathrm{H}_{7}^{+}$que muestra una disposición simétrica del protón entre las dos subunidades de $\mathrm{NH}_{3}$. b) estructura de equilibro asimétrica del $\mathrm{N}_{2} \mathrm{D}_{7}{ }^{+}$cuando se incluye el tratamiento cuántico de los núcleos

los espectros en fase gaseosa. Incluso los modelos mixtos, en los que se incluye específicamente la primera esfera de solvatación mediante la solvatación específica por un número reducido de moléculas de disolvente del compuesto que absorbe, mientras que el resto del disolvente se simula mediante un modelo continúo que depende de la constante dieléctrica del mismo, están lejos de producir resultados con precisión espectroscópica.

Los fenómenos de solvatación resultan críticos en otra pléyade de fenómenos entre los que merecen ser destacados las interacciones de aerosoles en las nubes y su impacto en el fenómeno del calentamiento global. De hecho aunque la nucleación para formar aerosoles atmosféricos ha recibido una gran atención en los últimos años (26-28), a lo que han contribuido notoriamente los cálculos quimicocuánticos, la formación de estas partículas está muy lejos de ser entendida a nivel molecular. Parece estar bien establecido que la nucleación comienza con la formación de complejos moleculares (véase Figura 7) con un tamaño típico del orden de $1 \mathrm{~nm}$; pero se desconocen las leyes que gobiernan el crecimiento de las gotas (29) mientras que la evaporación en estos microsistemas es muy difícil de modelizar.

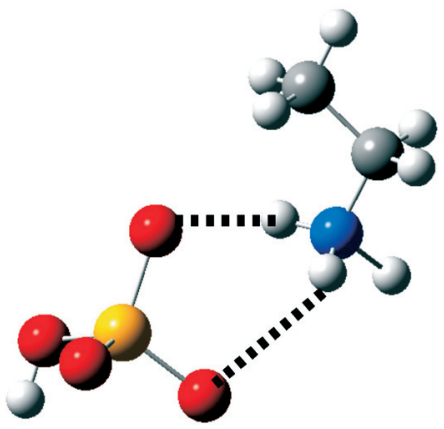

a
Los fenómenos de solvatación y otras muchas interacciones moleculares están gobernadas por interacciones débiles, que van desde las típicas fuerzas de dispersión hasta los enlaces de hidrógeno. De hecho fenómenos tan cotidianos como la capacidad de la salamanquesa para caminar por paredes verticales, está directamente relacionado con interacciones de van der Waals, al igual que las propiedades sorprendentes de resistencia de las telas de araña son consecuencia de la formación de enlaces de hidrógeno. Sin embargo, aunque la química cuántica actual es capaz de describir con extraordinaria precisión las energías de lo que solemos llamar enlaces químicos, esta precisión baja significativamente cuando se trata de interacciones débiles, entre otras razones porque la energía de interacción es tan pequeña, que aunque el error absoluto asociado con los cálculos quimicocuánticos sea similar al alcanzado en el tratamiento de enlaces químicos, el error relativo es mucho más grande. Por otra parte dado que las fuerzas de dispersión están omnipresentes en la naturaleza acompañan a otras interacciones débiles, como las interacciones dipolares, cuadrupolares o los ya mencionados enlaces de hidrógeno. Esto significa que una adecuada disección de las diferentes contribuciones es un reto to-

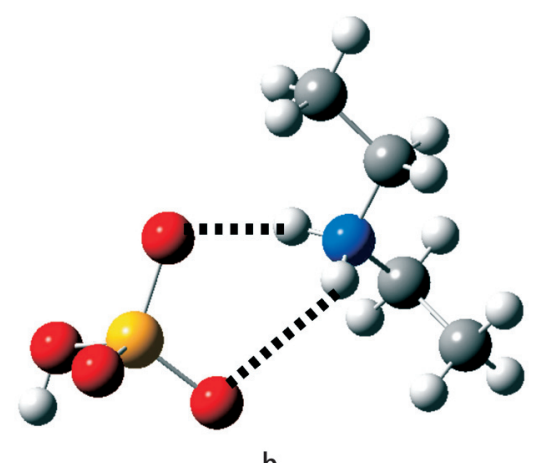

b

Figura 7. Estructura de complejos moleculares entre $\mathrm{H}_{2} \mathrm{SO}_{4}$ y a) metil amina, b) dimetil amina que juegan el papel de centros de nucleación en la generación de aerosoles atmosféricos. 
davía más difícil de alcanzar, ya que cada componente será más pequeño que la pequeña energía de interacción. Sin embargo recientemente se han desarrollado métodos potentes capaces de alcanzar precisión química incluso en aquellos casos en los que las fuerzas de London son un componente importante de la energía de interacción (30). Así, ya es posible calcular con precisión, por ejemplo, la energía de interacción entre sistemas aromáticos extendidos y fulerenos (véase Figura 8) donde la dispersión es el término dominante. Lo mismo puede decirse respecto a las interacciones metal-metal muy frecuentes en complejos organometálicos (31).

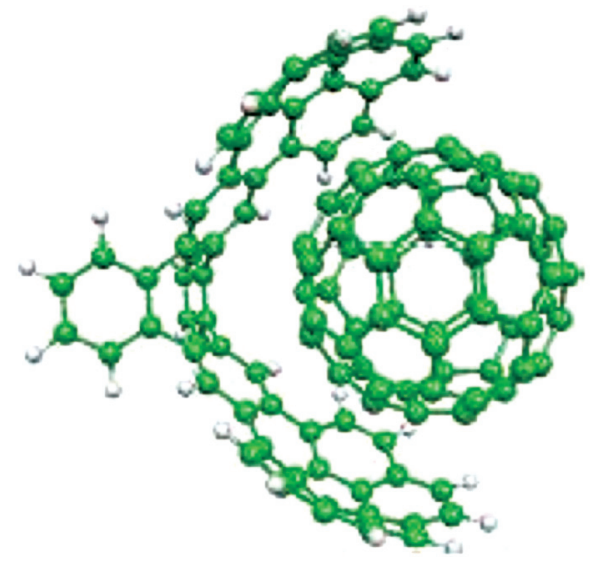

Figura 8. Complejo entre un sistema aromático extendido (buckyball atacher) y un fulereno, cuya energía de interacción ha sido calculada con precisión mediante métodos de la teoría del funcional de la densidad que incluyen términos dispersivos (30)

Pero donde sin duda la contribución de la química computacional es imprescindible es en la separación de los diferentes componentes de la energía de interacción ya que no existe un marco experimental adecuado para llevar a cabo tal disección, mientras que desde un punto de vista computacional resulta relativamente simple determinar cual es la contribución de cada componente de la interacción. Algunos resultados sorprendentes han visto la luz recientemente. Por brevedad citaremos sólo dos, dada su relevancia. Hasta no hace mucho la comunidad científica estaba convencida que las interacciones que mantenían unidas las hebras del ADN para formar la famosa hélice eran enlaces de hidrógeno entre las diferentes bases que las componen. Sin embargo, cálculos precisos recientes han llevado a la conclusión de que las fuerzas de dispersión asociada al apilamiento (staking) de los diferentes anillos a lo largo de las hélices, juegan un papel crucial (32), hasta el punto de que se apunta a que la ausencia de estas interacciones harian a la hélice de ADN inestable (33). El segundo ejemplo muestra como sorprendentemente la dispersión llega a dominar a la repulsión colombiana. Este es el caso en la formación de complejos de rutenio doblemente cargados (véase Esquema 1) donde dos fragmentos de ( $\mathrm{L})_{2} R u^{+}$ se unen a través de una interacción $R u-R u$ típicamente dispersiva para dar lugar a un complejo $\left[(\mathrm{L})_{2} \mathrm{Ru}-\mathrm{Ru}(\mathrm{L})_{2}\right]^{2+}$ estable frente a la explosión colombiana que lo disociaría en las dos subunidades (34).

2

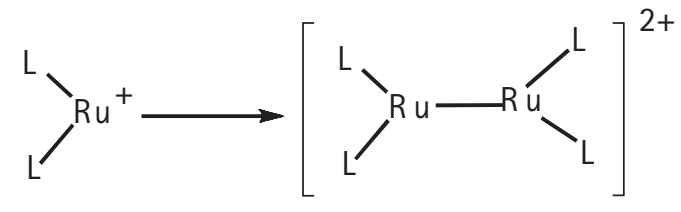

Esquema 1

Sin embargo son todavía muchos los retos que afrontar en el dominio de las interacciones débiles que gobiernan fenómenos fundamentales en química o bioquímica, como puede ser el plegamiento de las proteínas, que se ha podido modelizar en un buen número de casos, pero sobre cuyo mecanismo a nivel molecular aún no sabemos lo suficiente, o la interacción de moléculas con superficies, que juega un papel decisivo en los problemas de adsorción y por ende en la catálisis heterogénea. Son muchas las sorpresas que nos aguardan en este apasionante dominio como parecen apuntar resultados muy recientes, que indican (35) que en la interacción entre tetraciano-p-quinodimetano y superficies metálicas provocan una distorsión tanto de la molécula adsorbida como de la superficie metálica, pero lo que es más importante, estas modificaciones estructurales provocan nuevas interacciones intermoleculares asociadas a la tensión producida por la distorsión. Igualmente apasionante es la posibilidad de que la vida se haya originado por el efecto catalítico de superficies minerales en el ensamblaje de biomoléculas elementales para formar las macromoléculas esenciales de la vida. Diversos estudios teóricos recientes (36-38) en este campo parecen confirmar la hipótesis de J.D. Bernal que en 1949 sugirió que las superficies minerales en la naturaleza pueden adsorber e inmovilizar pequeñas moléculas bioquímicas y así catali- 
zar su condensación (39). De hecho, los modelos teóricos (véase Figura 9) confirman el papel catalítico que ejercen determinados aluminosilicatos en la formación de enlaces peptídicos (38). Es patente lo mucho que queda por hacer en esta dirección, pero los resultados ya obtenidos no pueden ser más motivadores, y quizás en un futuro no muy lejano seamos capaces de establecer con cierta certidumbre el mecanismo detrás del origen de la vida.
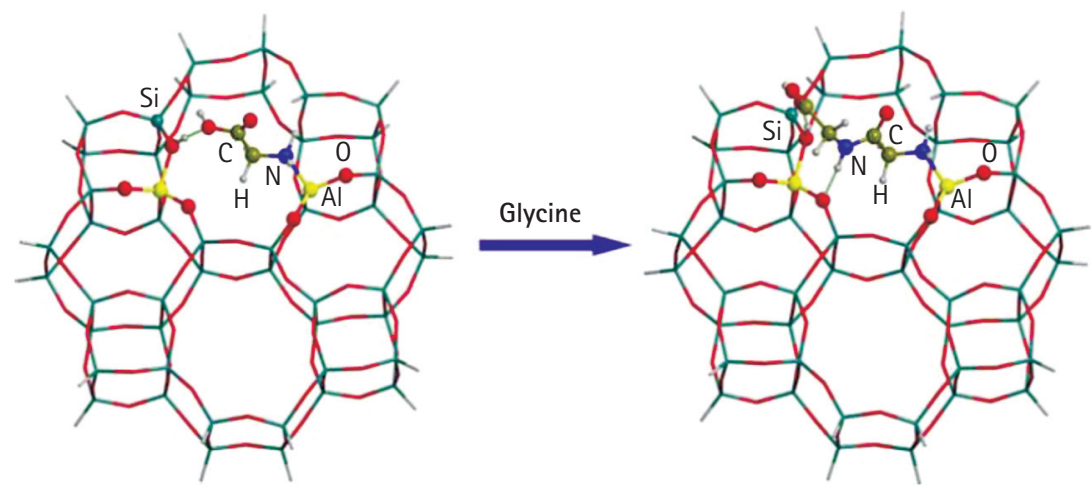

Figura 9. Modelo usado para el estudio de la posible formación de enlaces peptídicos sobre la superficie de aluminosilicatos (38)

No sólo el origen de la vida espera y precisa de las contribuciones de la química computacional, pero también su preservación. Uno de los tópicos que por razones que al lector no se le escapan, ha recibido mayor atención en la última década es el daño por radiación (radiation damage) y una vez más los mecanismos que desencadenan estos daños sólo podrán ser bien establecidos a nivel molecular con el concurso de la química computacional. Parece bien establecido que estos daños son producidos por radicales, pero existen cada vez más evidencias de que pueden ser también originados por electrones libres, y aquí nace ya un nuevo reto para el futuro ya que el estudio de los aniones de ADN es todo un serio problema para los modelos teóricos, al igual que lo es el estudio de los procesos de asociación disociativa que seguiría a la interacción de electrones de muy baja energía con moléculas de ADN.

En párrafos precedentes hemos hablado de fenómenos de astroquímica, que ocurren en entornos con situaciones límites de baja presión y baja temperatura, pero hay todo un mundo mucho más próximo por explorar cuando uno se va al otro extremo, al de las altas presiones, como las que se dan en el interior de nuestro planeta. En el régimen de muy altas presiones todos nuestros conceptos de enlace y de estructura molecular comienzan a crujir lastimeramente. Dos ejemplos paradigmáticos ilustran bien los dramáticos cambios que la presión induce en un sistema. Uno de ellos es el comportamiento del hidrógeno molecular bajo condiciones de alta presión. A presión normal el hidrógeno es una gas no muy le- jano a la idealidad, como demuestra su bajo punto de fusión (14 K), sin embargo cálculos recientes (40) indican que a un presión de $300 \mathrm{GPa}$ se convierte en un metal molecular antes de transformarse en un metal alcalino. El segundo ejemplo lo constituye el silano $\left(\mathrm{SiH}_{4}\right)$ que en condiciones normales de presión presenta un silicio tetracoordinado, mientras que a presiones de $160 \mathrm{GPa}$ aparece coordinado a ocho átomos de hidrógeno (41), tal y como muestra la Figura 10.

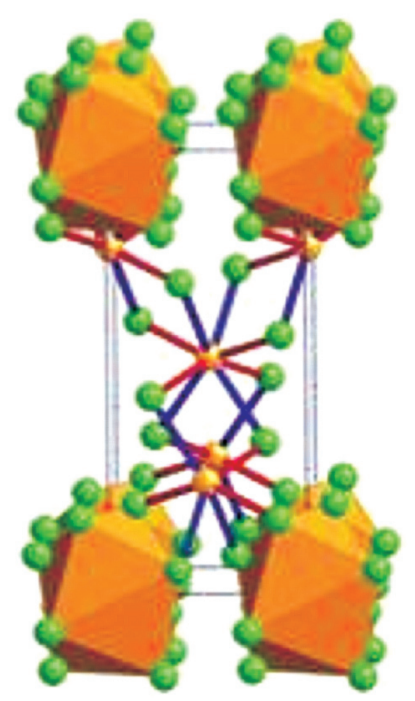

Figura 10. Estructura del $\mathrm{SiH}_{4}$ bajo presión de 160 GPa. En ella se puede observar que el átomo de silicio (naranja) aparece octacoordinado formando cuatro pares de puentes de hidrógeno (verde) del tipo de los que aparecen en el diborano (41) 
Los estudios teóricos sobre sistemas en el rango de altas presiones son relativamente recientes y por ello estamos muy lejos de entender los cambios que se promueven en la estructura electrónica de los sistemas y en su enlace, aunque los primeros modelos apuntan a que hay que estar preparados para admitir que la presión puede provocar la ocupación de orbitales que nunca se nos ocurriria que pudiesen participar en la estructura electrónica de un sistema químico (41). De hecho, la posible naturaleza metálica del $\mathrm{H}_{2}$ bajo alta presión, a pesar de ser el sistema más simple, no está libre de controversia, ya que medidas ópticas realizadas a $320 \mathrm{GPa}$ indican que por encima de $300 \mathrm{GPa}$ el hidrógeno sólido muestra evidencias de la existencia de un bandgap electrónico (42), que, en contra con las predicciones teóricas, indicaría un comportamiento semiconductor, que sólo convergería a un comportamiento puramente metálico a aproximadamente $450 \mathrm{GPa}$.

Una gran mayoría de los compuestos conocidos contienen átomos de los dos primeros períodos, desde el $\mathrm{H}$ al $\mathrm{Cl}$, pero el universo de los elementos de los últimos períodos es también apasionante, esencialmente porque sus electrones se mueven a velocidades relativistas. Aunque en los átomos ligeros los efectos relativistas son casi despreciables, ya que dichos efectos crecen con $Z^{2}$, en los elementos pesados llegan a determinar las propiedades fisico-químicas de los mismos. El color del oro tiene este origen y la eficacia de las baterías de plomo también, ya que $1.8 \mathrm{~V}$ del total de los $2.1 \mathrm{~V}$ que marcan el voltaje estándar de estas baterías tiene su origen en efectos relativistas. Los efectos relativistas contraen los enlaces químicos y son la fuente de una saga de nuevas especies, como los complejos endohédricos, entre los que el Pu@ $\mathrm{Pb}_{12}$ (véase Figura 11) es un paradigmático ejemplo, En este sistema el átomo de Pu presenta una corteza externa de 32 electrones, en la que se encuentra completamente ocupados los orbitales $7 s, 7 p, 6 d$ y $5 f$ que interaccionan con un orbital $6 p$ de la caja de $\left[\mathrm{Pb}_{12}\right]^{2-}$. Este hallazgo ha permitido definir para los actínidos una nueva regla, la regla de 32 electrones (43), análoga a la de 18 electrones que gobierna la estabilidad de complejos de metales de transición. De hecho, cálculos teóricos de teoría del funcional de la densidad han demostrado que nuevos complejos, en los que la caja es un cluster de 28 átomos de carbono encerrando un actínido, $A n @ C_{28}\left(A n=T h, P^{+}\right.$, $\left.\mathrm{U}^{2+}, \mathrm{Pu}^{4+}\right)$, presentan una notable estabilidad y cumplen el principio de 32 electrones (44).

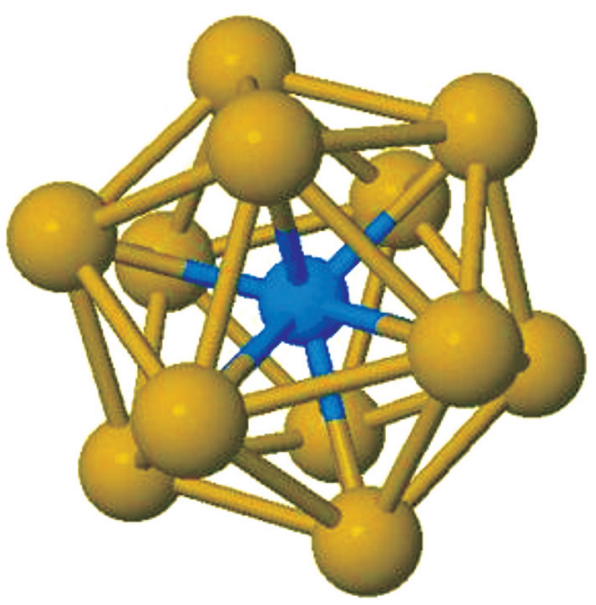

Figura 11. Estructura del complejo endohédrico Pu@Pb ${ }_{12}$.En azul el átomo de Pu y en amarillo los átomos de Pb. Ilustración tomada de la ref (43)

Toda una pléyade de nuevas especies de átomos pesados están esperando ser sintetizados y analizados mediante diferentes técnicas, planteando nuevos retos a la interpretación de su estabilidad y forzando a la generalización de reglas bien conocidas como la aludida regla de los 18 electrones, que permite predecir la estabilidad de complejos de metales de transición, pero que necesita ser extendida a una nueva regla, la de los 32 electrones, para poder explicar la estabilidad de complejos de actínidos. Una inmensa tarea queda por hacer si queremos entender el enlace en estas nuevas especies, y el papel que los efectos relativistas juegan en todos ellos.

Cerramos aquí esta breve presentación de retos que se plantean para el inmediato futuro conscientes de que sólo una mínima parte de ellos ha sido discutida aquí, y estamos convencidos de que muchos lectores habrian hecho una selección distinta e igualmente apasionante. Temas como las nano-lentes $(45,46)$, la capacidad catalítica de determinadas nanopartículas $(47,48)$, la generación de dispositivos fotovoltaicos todo orgánicos (49-51), la química de los clusters de carbono $(52,53)$, de los fulerenos $(54,55)$ y de los nanotubos de carbono $(56,57)$ o de otros elementos $(58,59)$, la existencia de nuevas estructuras como los carbones, con átomos de carbono divalentes descritos por G. Frenking (60), las especies múltiplemente cargadas (61-63), la posibilidad de diseñar 
fármacos $(64,65)$ o nuevos materiales con propiedades especificas (66) y un sinfín de otros tópicos definen un futuro apasionante para cualquiera que desee enredarse hasta la médula en la búsqueda de las razones últimas que explican las singulares propiedades de estos y otros muchos sistemas. Como acertadamente dijo hace ya unos cuantos años el genial Richard P. Feynman, there is plenty of room at the bottom (67).

\section{REFERENCES}

(1) Moller, C.; Plesset, M. S., Phys. Rev., 1934, 46, 0618.

(2) Foster, J. M.; Boys, S. F., Rev. Mod. Phys., 1960, 32, 305.

(3) Herzberg, G., Proc. R. Soc. London Ser. A 1961, 262, 291.

(4) Harrison, J. F.; Allen, L. C., J. Am. Chem. Soc., 1969, 91, 807.

(5) Bender, C. F.; Schaefer, H. F., J. Am. Chem. Soc., 1970, 92, 4985.

(6) Bernheim, R. A.; Bernard, H. W.; Wang, P. S.; Wood, L. S.; Skell, P. S., J. Chem. Phys., 1970, 53, 1280.

(7) Wasserman, E.; Yager, W. A.; Kuck, V. J., Chem. Phys. Lett., 1970, 7, 409.

(8) Herzberg, G.; Johns, J. W. C., J. Chem. Phys., 1971, 54, 2276.

(9) Curtiss, L. A.; Raghavachari, K.; Trucks, G. W.; Pople, J. A., J. Chem. Phys., 1991, 94, 7221.

(10) Curtiss, L. A.; Raghavachari, K.; Redfern, P. C.; Rassolov, V.; Pople, J. A., J. Chem. Phys., 1998, 109, 7764.

(11) Hohenberg, P.; Kohn, W., Phys. Rev. B 1964, 136, B864.

(12) Kohn, W.; Sham, L. J., Phys. Rev., 1965, 140, 1133.

(13) Tennyson, J.; Bernath, P. F.; Brown, L. R.; Campargue, A.; Carleere, M. R.; Csaszar, A. G.; Gamache, R. R.; Hodges, J. T.; Jenouvrier, A.; Naumenko, O. V.; Polyansky, O. L.; Rothman, L. S.; Toth, R. A.; Vandaele, A. C.; Zobov, N. F.; Daumont, L.; Fazliev, A. Z.; Furtenbacher, T.; Gordon, I. E.; Mikhailenko, S. N.; Shirin, S. V., J. Quant. Spectrosc. Radiat. Transf., 2009, 110, 573.
(14) Tennyson, J.; Bernath, P. F.; Brown L. R.; Campargue, A.; Csaszar, A. G.; Daumont, L.; Gamache, R. R.; Hodges, J. T.; Naumenko, O. V.; Polyansky, O. L.; Rothman, L. S.; Toth, R. A.; Vandaele, A. C.; Zobov, N. F.; Fally, S.; Fazliev, A. Z.; Furtenbacher, T.; Gordon, I. E.; Hu, S. M.; Mikhailenko, S. N.; Voronin, B. A., J. Quant Spectrosc. Radiat. Transf., 2010, 111, 2160.

(15) Czako, G.; Matyus, E.; Simmonett, A. C.; Csaszar, A. G.; Schaefer, H. F.; Allen, W. D., J. Chem. Theory Comput., 2008, 4, 1220.

(16) Sofia, N., http://www.nasa.gov/mission_pages/SOFIA/index.html, 2010.

(17) Boersma, C.; Bauschlicher, C. W.; Allamandola, L. J.; Ricca, A.; Peeters, E.; Tielens, A., Astron Astrophys., 2010, 511, 16.

(18) Ricca, A.; Bauschlicher, C. W.; Mattioda, A. L.; Boersma, C.; Allamandola, L. J., Astrophys. J., 2010, 709, 42.

(19) Bauschlicher, C. W.; Boersma, C.; Ricca, A.; Mattioda, A. L.; Cami, J.; Peeters, E.; de Armas, F. S.; Saborido, G. P.; Hudgins, D. M.; Allamandola, L. J., Astrophys. J. Suppl. Ser., 2010, 189, 341.

(20) Schreiner, P. R.; Reisenauer, H. P.; Matyus, E.; Csaszar, A. G.; Siddiqi, A.; Simmonett, A. C.; Allen, W. D., Phys. Chem. Chem. Phys., 2009, 11, 10385.

(21) Matyus, E.; Fabri, C.; Szidarovszky, T.; Czako, G.; Allen, W. D.; Csaszar, A. G., J. Chem. Phys., 2010, 133, 14.

(22) Trefler, M.; Gush, H. P., Phys. Rev. Lett., 1968, 20, 703. 
(23) González, S. A.; Aguirre, N. F.; Reyes, A., Int. J. Quantum Chem., 2008, 108, 1742.

(24) González, S. A.; Reyes, A., Int. J. Quant. Chem., 2010, 110, 689.

(25) Reyes, A., Including Nuclear Quantum effects in Convetional Quantum Chemistry Methods, 2010. Comunicación al $36^{\text {ème }}$ CHITEL, Anglet, France.

(26) Kurten, T.; Vehkamaki, H., "Investigating atmospheric sulfuric acidwater-ammonia particle formation using quantum chemistry", in $\mathrm{Ad}$ vances in Quantum Chemistry, Vol 55: Applications of Theoretical Methods to Atmospheric Science, Elsevier Academic Press Inc, San Diego, 2008, Vol. 55, pp. 407.

(27) Kurten, T.; Loukonen, V.; Vehkamaki, H.; Kulmala, M., Atmos. Chem. Phys., 2008, 8, 4095.

(28) Zhao, J.; Khalizov, A.; Zhang, R. Y.; McGraw, R., J. Phys. Chem. A, 2009, 113,680

(29) Gross, A.; Nielsen, 0. J.; Mikkelsen, K. V., "From molecules to droplets", in Advances in Quantum Chemistry, Vol 55: Applications of Theoretical Methods to Atmospheric Science, Elsevier Academic Press Inc, San Diego, 2008, Vol. 55, pp. 355.

(30) Grimme, S.; Antony, J.; Ehrlich, S.; Krieg, H., J. Chem. Phys., 2010, 132, 19.

(31) Schwabe, T.; Grimme, S.; Djukic, J. P., J. Am. Chem. Soc., 2009, 131, 14156.

(32) Rezac, J.; Hobza, P., Chem.-Eur. J., 2007, 13, 2983.

(33) Cerny, J.; Kabelac, M.; Hobza, P., J. Am. Chem. Soc., 2008, 130, 16055.

(34) Grimme, S., "Computationally Efficient and Accurate Dispersion Correction for Density Functional Theory", 2010. Conferencia impartida en el Congreso sobre Molecu- lar Quantum Mechanics: From Methylene to DNA and Beyond.

(35) Tseng, T. C.; Urban, C.; Wang, Y.; Otero, R.; Tait, S. L.; Alcami, M.; Ecija, D.; Trelka, M.; Gallego, J. M.; Lin, N.; Konuma, M.; Starke, U.; Nefedov, A.; Langner, A.; Woll, C.; Herranz, M. A.; Martin, F.; Martin, N.; Kern, K.; Miranda, R., Nature Chem., 2010, 2, 374.

(36) Rimola, A.; Sodupe, M.; Tosoni, S.; Civalleri, B.; Ugliengo, P., Langmuir, 2006, 22, 6593.

(37) Rimola, A.; Sodupe, M.; Ugliengo, P., J. Phys. Chem. C, 2009, 113, 5741.

(38) Ugliengo, P.; Rimola, A.; Sodupe, M., Orig. Life Evol. Biosph., 2009, 39, 197.

(39) Bernal, J. D., Proc. Phys. Soc. London A, 1949, 62, 537.

(40) Johnson, K.; Ashcroft, N. W., Nature, 2000, 403, 632.

(41) Grochala, W.; Hoffmann, R.; Feng, J.; Ashcroft, N. W. Angew. Chem. Int. Ed., 2007, 46, 3620.

(42) Loubeyre, P.; Occelli, F.; LeToullec, R. Nature 2002, 416, 613.

(43) Dognon, J. P.; Clavaguera, C.; Pyykko, P. Angew. Chem. Int. Ed., 2007, 46, 1427.

(44) Dognon, J. P.; Clavaguera, C.; Pyykko, P. J. Am. Chem. Soc., 2009, 131, 238.

(45) Lee, J. Y.; Hong, B. H.; Kim, W. Y.; Min, S. K.; Kim, Y.; Jouravlev, M. V.; Bose, R.; Kim, K. S.; Hwang, I. C.; Kaufman, L. J.; Wong, C. W.; Kim, P. Nature 2009, 460, 498.

(46) Kim, W. Y.; Choi, Y. C.; Min, S. K.; Cho, Y.; Kim, K. S. Chem. Soc. Rev., 2009, 38, 2319.

(47) Park, J. B.; Graciani, J.; Evans, J.; Stacchiola, D.; Senanayake, S. D.; Barrio, L.; Liu, P.; Sanz, J. F.; Hrbek, J.; Rodriguez, J. A. J. Am. Chem. Soc., 2010, 132, 356.
(48) Migani, A.; Vayssilov, G. N.; Bromley, S. T.; Illas, F.; Neyman, K. M. Chem. Commun., 2010, 46, 5936.

(49) Medina, A. S.; Claessens, C. G.; Rahman, G. M. A.; Lamsabhi, A. M.; Mó 0.; Yáñez, M.; Guldi, D. M.; Torres, T. Chem. Commun., 2008, 1759.

(50) Garcia-Belmonte, G.; Boix, P. P.; Bisquert, J.; Lenes, M.; Bolink, H. J.; La Rosa, A.; Filippone, S.; Martin, N. J. Phys. Chem. Lett., 2010, 1, 2566.

(51) Costa, R. D.; Fernandez, G.; Sanchez, L.; Martin, N.; Orti, E.; Bolink, H. J. Chem.-Eur. J., 2010, 16, 9855.

(52) Martinet, G.; Diaz-Tendero, S.; Chabot, M.; Wohrer, K.; Della Negra, S.; Mezdari, F.; Hamrita, H.; Desesquelles, P.; Le Padellec, A.; Gardes, D.; Lavergne, L.; Lalu, G.; Grave, X.; Clavelin, J. F.; Hervieux, P. A.; Alcami, M.; Martin, F. Phys. Rev. Lett., 2004, 93, 4.

(53) Diaz-Tendero, S.; Sanchez, G.; Alcami, M.; Martin, F.; Hervieux, P. A.; Chabot, M.; Martinet, G.; Desesquelles, P.; Mezdari, F.; WohrerBeroff, K.; Della Negra, S.; Hamrita, H.; Le Padellec, A.; Montagnon, L. Int. J. Mass Spectrom., 2006, 252, 126.

(54) Rentenier, A.; Ruiz, L. F.; Diaz-Tendero, S.; Zarour, B.; Moretto-CapeIle, P.; Bordenave-Montesquieu, D.; Bordenave-Montesquieu, A.; Hervieux, P. A.; Alcami, M.; Politis, M. F.; Hanssen, J.; Martin, F. Phys. Rev. Lett., 2008, 100, 4.

(55) Zettergren, H.; Wang, Y.; Lamsabhi, A.; Alcami, M.; Martin, F., J. Chem. Phys., 2009, 130, 6.

(56) Chen, Z. F.; Nagase, S.; Hirsch, A.; Haddon, R. C.; Thiel, W.; Schleyer, P. V. Angew. Chem. Int. Edit., 2004, 43, 1552.

(57) Dukovic, G.; White, B. E.; Zhou, Z. Y.; Wang, F.; Jockusch, S.; Steigerwald, M. L.; Heinz, T. F.; Friesner, R. A.; 
Turro, N. J.; Brus, L. E. J. Am. Chem. Soc., 2004, 126, 15269.

(58) Oger, E.; Crawford, N. R. M.; Kelting, R.; Weis, P.; Kappes, M. M.; Ahlrichs, R. Angew. Chem. Int. Edit., 2007, 46, 8503.

(59) Riikonen, S.; Foster, A. S.; Krasheninnikov, A. V.; Nieminen, R. M. Phys. Rev. B 2009, 80, 14.

(60) Frenking, G.; Tonner, R. Pure Appl. Chem., 2009, 81, 597.

(61) Palacios, A.; Martín, F.; Mó, 0.; Yáñez, M.; Maksic, Z. B. Phys. Rev. Lett., 2004, 92, 133001.

(62) Trujillo, C.; Lamsabhi, A. M.; Mó, 0.; Yáñez, M. Phys. Chem. Chem. Phys., 2008, 10, 3229.
(63) Corral, I.; Trujillo, C.; Salpin, J. Y.; Yáñez, M., "Ca2+ reactivity in the gas phase. Bonding, catalytic effects and coulomb explosions", in Challenges and Advances in Computational Chemistry and Physics. Vol. 12 Kinetics and Dynamics: From Nano- to Bio-Scale, Paneth, P., Dybala-Defratyka, A., Eds., Springer, London, 2010, Vol. 12

(64) Kelly, J. X.; Smilkstein, M. J.; Brun, R.; Wittlin, S.; Cooper, R. A.; Lane, K. D.; Janowsky, A.; Johnson, R. A.; Dodean, R. A.; Winter, R.; Hinrichs, D. J.; Riscoe, M. K., Nature, 2009, 459, 270.
(65) Bax, B. D.; Chan, P. F.; Eggleston, D. S.; Fosberry, A.; Gentry, D. R.; Gorrec, F.; Giordano, I.; Hann, M. M.; Hennessy, A.; Hibbs, M.; Huang, J. Z.; Jones, E.; Jones, J.; Brown, K. K.; Lewis, C. J.; May, E. W.; Saunders, M. R.; Singh, 0.; Spitzfaden, C. E.; Shen, C.; Shillings, A.; Theobald, A. J.; Wohlkonig, A.; Pearson, N. D.; Gwynn, M. N., Nature, 2010, 466, 935.

(66) Coronado, E.; Galán-Mascaros, J. R.; Gómez-García, C. J.; Laukhin, V., Nature, 2000, 408, 447.

(67) Feynman, R. P., There's plenty of room at the bottom. An Invitation to Enter a NewField of Physics. 